\title{
Targeted molecular imaging of TLR4 in hepatocellular carcinoma using zwitterionic near-infrared fluorophores
}

\author{
Yuanyuan Ji ${ }^{1,2 \#}$, Zhidong Wang ${ }^{1,2 \#}$, Kai Bao ${ }^{2}$, G. Kate Park ${ }^{2}$, Homan Kang ${ }^{2}$, Shuang $\mathrm{Hu}^{2}$, \\ Eric McDonald ${ }^{2}$, Moon Suk Kim ${ }^{3}$, Satoshi Kashiwagi ${ }^{2}$, Hak Soo Choi ${ }^{2}$ \\ ${ }^{1}$ Scientific Research Centre and Department of VIP General Surgery, The Second Affiliated Hospital, Xi'an Jiaotong University, Xi'an 710004, \\ China; ${ }^{2}$ Gordon Center for Medical Imaging, Department of Radiology, Massachusetts General Hospital and Harvard Medical School, Charlestown, \\ MA, USA; ${ }^{3}$ Department of Molecular Science and Technology, Ajou University, Suwon, Korea \\ "These authors contributed equally to this work.
}

Correspondence to: Satoshi Kashiwagi. Gordon Center for Medical Imaging, Department of Radiology, Massachusetts General Hospital and Harvard Medical School, 149 13th Street, Charlestown, MA 02129, USA. Email: skashiwagi@mgh.harvard.edu; Moon Suk Kim. Department of Molecular Science and Technology, Ajou University, Suwon, 16499, Korea. Email: moonskim@ajou.ac.kr; Hak Soo Choi. Gordon Center for Medical Imaging, Department of Radiology, Massachusetts General Hospital and Harvard Medical School, 149 13th Street, Charlestown, MA 02129, USA. Email: hchoi12@mgh.harvard.edu.

Background: Tumor-associated macrophages (TAMs) are one of the most abundant immune cell types in solid tumors and implicated in tumor progression. Toll-like receptor 4 (TLR4) is expressed in TAMs and plays a key role in immune surveillance and tumor progression. Therefore, molecular imaging of TLR4 has potential not only for detection of TAM-enriched progressing tumors, but also evaluation of TLR4 expression in tumor microenvironment.

Methods: Here, we report that near-infrared (NIR) fluorescence imaging can provide a real-time imaging of a syngeneic model of murine hepatocellular carcinoma using targeted strategy against TLR4. We conjugated a zwitterionic NIR fluorophore ZW800-1C with minimal nonspecific tissue interactions to anti-TLR4 antibody and observed its targetability. The bioconjugates showed high affinity to murine macrophages in cell culture and in vivo.

Results: Interestingly, we observed predominant NIR signals in the tumor site, which persisted for more than $48 \mathrm{~h}$ after single intravenous administration of the bioconjugate.

Conclusions: This result suggests that TLR4 targeting combined with NIR fluorescence imaging is a useful tool for cancer imaging. This imaging strategy could be used to detect cancerous tissue with the increased TAM content and evaluate the status of TLR4 signaling in solid tumors, ultimately impacting on the diagnostic and prognostic imaging of human cancers.

Keywords: Zwitterionic fluorophore; NIR imaging; toll-like receptor 4; hepatocellular carcinoma; tumorassociated macrophages (TAMs)

Submitted Aug 14, 2019. Accepted for publication Sep 03, 2019.

doi: 10.21037/qims.2019.09.04

View this article at: http://dx.doi.org/10.21037/qims.2019.09.04

\section{Introduction}

Growing evidence has demonstrated the importance of host immune system in tumor progression (1). Amongst the immune cells involved in this process, tumor-associated macrophages (TAMs) are predominantly abundant in solid tumors and have gained considerable attention because of their critical roles in tumor progression $(2,3)$. Tolllike receptors (TLRs) belong to pathogen recognition 
receptors and trigger intracellular signaling cascades in response to pathogens to evoke the secretion of interferons and proinflammatory cytokines, resulting in activating host defense programs necessary for innate or adaptive immune responses (4). TLR4 is one of the most studied TLRs expressed in the tumor microenvironment and widely implicated in its key role in immune surveillance and tumor progression (5). Molecular imaging of TLR4 therefore represents the potential for diagnostic imaging of tumor progression as well as functional evaluation of TLR4 signaling in solid tumors.

Liver cancer is one of the most common cancers worldwide and hepatocellular carcinoma (HCC) is by far the most common type of primary liver cancer (6). HCC features an inflammatory background and innate immunity closely related to the HCC carcinogenesis (6). TLR4 has been indicated to facilitate the recruitment of regulatory T-cells and to promote intrahepatic metastasis through its interaction with macrophages (7). Ablation of TLR4 signaling is considered to be a promising strategy to combat HCC by reducing liver inflammation and tumorigenesis (8). Thus, molecular imaging of TLR4 would be a useful tool to design or optimize therapeutic strategies against HCC.

Here we report a robust strategy for molecular imaging of TLR4 using a zwitterionic targeted molecule, anti-TLR4 antibody conjugated with nonsticky ZW800-1C, which provides minimum to no serum binding and nonspecific tissue background, thus enabling sensitive, specific, and real-time imaging of liver cancer in a mouse model $(9,10)$ and has excellent optical properties and high serum stability in physiological environment (11).

\section{Methods}

\section{Bioconjugation of ZW800-1C on anti-TLR4 antibody}

The NHS ester form of ZW800-1C (11-13) was conjugated with TLR4/MD-2 complex monoclonal antibody (MTS510, Thermo-Fisher Scientific, MA) as previously described (10). Briefly, $20 \mathrm{nmol}$ of ZW800-1C NHS ester was added to $1 \mathrm{nmol}$ of anti-TLR4 antibody and incubated at room temperature for $3 \mathrm{~h}$ in PBS, $\mathrm{pH} 7.8$. The anti-TLR4 antibody-ZW800-1C (TLR4-ZW800) conjugates were then purified using a mini Bio-Gel P-6 desalting column (Bio-Rad, Hercules, CA) and concentrated with a 10,000 molecular-weight cutoff (MWCO) spin column (Vivaspin 500, 10K MWCO). The labeling ratio was calculated based on the Beer-Lambert law by determining the concentration of each compound. Extinction coefficients for the antibody was $210,000 \mathrm{M}^{-1} \mathrm{~cm}^{-1}$ measured at $280 \mathrm{~nm}$, and ZW800-1C was $120,000 \mathrm{M}^{-1} \mathrm{~cm}^{-1}$ at $765 \mathrm{~nm}$, respectively. The TLR4ZW800 was then analyzed for the optical properties using a UV-Vis-NIR spectrophotometer (USB-ISS-UV/VIS, Ocean Optics, FL).

\section{Cell labeling with TLR4-ZW800}

RAW264.7 cells were purchased from ATCC (Manassas, VA) and grown in DMEM (Mediatech, Hermdon, VA) supplemented with $10 \%$ FBS and gentamicin $(50 \mathrm{mg} / \mathrm{mL})$ in a humidified incubator at $37{ }^{\circ} \mathrm{C}$ under $5 \% \mathrm{CO}_{2}$ in air. For labeling with TLR4-ZW800, cells were incubated at $37^{\circ} \mathrm{C}$ for $60 \mathrm{~min}$ in the presence of $2 \mu M$ TLR4-ZW800. For imaging analysis of specific binding of TLR4-ZW800 to the cells, RAW264.7 cells were seeded onto sterilized glass coverslips in 24 -well plates $\left(1 \times 10^{5}\right.$ cells per well), incubated with TLR4-ZW800, and observed on a 4-channel NIR fluorescence microscope with two custom filter sets (TE2000U, Nikon, Japan) and CCD camera (C4742-8012AG, Hamamatsu Photonics, Japan) after gentle washing 3 times with 10\% FBS DMEM.

\section{Animal models and intraoperative imaging}

Six-week-old male C57BL/6 mice (stock \#000664) were purchased from the Jackson Laboratory (Bar Harbor, ME) and housed in an AAALAC-certified facility at Massachusetts General Hospital (MGH). All animal procedures were performed in accordance with the Public Health Service Policy on Humane Care of Laboratory Animals and approved by the MGH IACUC (protocol \#2016N000529). Mice were maintained under anesthesia by intraperitoneally injection with $100 \mathrm{mg} / \mathrm{kg}$ ketamine and $10 \mathrm{mg} / \mathrm{kg}$ xylazine (Webster Veterinary, Fort Devens, MA) for the entire duration of the experiment. The end of the tail was cut to be enable blood extraction. Before injection, blood was sampled in heparinized capillary tubes (Fisher Scientific, Pittsburgh, PA) as a reference and collected blood was stored in an ice box to prevent clotting. Mice were then injected with $10 \mathrm{nmol}$ of each TLR4-ZW800 in saline containing $5 w t / v \%$ BSA and blood was sampled at the following time points $(1,3,5,10$, $30,60,120,180$, and $240 \mathrm{~min})$ to estimate distribution $\left(t_{1 / 20}\right)$ and elimination $\left(t_{1 / 2 \beta}\right)$ blood half-life values. The collected blood samples were centrifuged for $20 \mathrm{~min}$ at $1,000 \mathrm{~g}$ in order to separate serum and blood plasma, and supernatants were then filled into capillary microtubes. Fluorescence 
intensities of the microtubes were measured using the inhouse built NIR imaging system. Results were presented as a bi-exponential decay curve using Prism version 8 software (GraphPad, San Diego, CA). For biodistribution study, mice were imaged using the in-house built real-time intraoperative NIR imaging system. A $760 \mathrm{~nm}$ excitation laser source $(4 \mathrm{~mW} / \mathrm{cm})$ was used with white light $(400-650 \mathrm{~nm}$; 40,000 lux). Color and NIR fluorescence images were acquired simultaneously with customized software at rates of up to $15 \mathrm{~Hz}$ over a field of view with $15 \mathrm{~cm}$ in diameter. After $4 \mathrm{~h}$ post-injection, mice were sacrificed to image organs and collected urine from the bladder. At least 3 mice were analyzed for each sample.

\section{In vivo tumor targeting}

To establish a syngeneic murine model of liver cancer, Hepa1-6 cells (ATCC) were cultured in DMEM with 5\% FBS and 100 units $/ \mathrm{mL}$ of penicillin and streptomycin. C57BL/6 mice were then inoculated subcutaneously with $1 \times 10^{7}$ Hepa1- 6 cells suspended in $150 \mu \mathrm{L}$ of saline/ Matrigel $(50 \mathrm{v} / \mathrm{v} \%)$ at the right flank. Once the long axis of the tumor diameter reached at a size of $0.5 \mathrm{~cm}, 10 \mathrm{nmol}$ of the TLR4-ZW800 conjugate in saline containing 5\% BSA was injected through tail vein. For real-time NIR fluorescence imaging, the real-time intraoperative FLARE imaging system was used (14). Briefly, tumor-bearing mice were anesthetized with isoflurane and imaged using a prism based multispectral CCD camera (JAI A/S, Denmark) 1, 2, 4, 6, 24, 48 and $72 \mathrm{~h}$ after injection under $760 \mathrm{~nm}$ excitation laser. Alternatively, mice were injected intravenously with $1 \times 10^{5}$ TLR4-ZW800-labeled RAW264.7 cells in $100 \mu \mathrm{L}$ saline and imaged at 1, 2, 4, 6, 24, 48 h post-injection. For histology, fluorescence microscopy was performed on a Nikon TE2000 with two custom filter sets (Chroma Technology, Brattleboro, VT). Upon completion of imaging, mice were euthanized and major organs were dissected for fluorescence imaging and histological evaluations.

\section{Quantitative analysis of fuorescence images}

The fluorescence and background intensities of a region of interest over each tissue were quantified using ImageJ v1.51 (National Institutes of Health, Bethesda, MD). The SBR was calculated as follows:

$$
S B R=\frac{I_{R O I}}{I_{\text {Auto }}}
$$

where $I_{R O I}$ denotes the average intensity of an ROI and $I_{\text {Auto }}$ represents the intensity of the muscle. The TBR was calculated using the same formula and $I_{T}$ representing the intensity of the tumor tissue.

$$
T B R=\frac{I_{T}}{I_{\text {Auto }}}
$$

\section{NIR fluorescence microscopy of tumor sections}

Excised liver tumors were trimmed and embedded in OCT compound. Ten- $\mu$ m thickness of frozen sections were cut by a cryostat (Leica, Germany). A part of the serial sections was stained with hematoxylin and eosin (H\&E). Fluorescence and bright-field images were acquired on the NIR fluorescence microscope and CCD camera.

\section{Statistical analysis}

A one-way ANOVA followed by Tukey's multiple comparisons tests was performed on Prism 8 software (GraphPad, San Diego, CA). Results were presented as mean \pm standard deviation (SD) for all the image analyses on the FLARE system and fluorescence microscopy.

\section{Results and discussion}

As shown in Figure 1A, the $\mathrm{N}$-hydroxysuccinimide (NHS) ester form of ZW800-1C was used to yield bioconjugated anti-TLR-4 antibody (TLR4-ZW800; Figure 1B). After purification using a membrane filtration column, strong NIR fluorescence was detectable from ZW800-1C under the real-time imaging system (Figure 1C) (15). The labeling ratio of fluorophores on antibody was determined to be 2.47 calculated by absorbance measurements of anti-TLR4 antibody at $280 \mathrm{~nm}(0.43)$ and ZW800-1C at $760 \mathrm{~nm}(1.04)$ based on the Beer-Lambert Law (Figure 1D).

To validate the specific binding of TLR4-ZW800 on the surface of living cells we used RAW264.7 cells, an established mouse macrophage cell line reported to constitutively express TLR4 (16). After $1 \mathrm{~h}$ incubation at $37^{\circ} \mathrm{C}$, strong NIR fluorescence signals were observed in the cytosol of RAW264.7 cells, indicating receptor-mediated endocytosis (Figure S1). We next determined the pharmacokinetics of TLR4-ZW800 in animal models. $10 \mathrm{nmol}$ of TLR4ZW800 was injected intravenously into C57BL/6 mice and blood was subsequently collected over the period of $4 \mathrm{~h}$ using heparinized capillary tubes, followed by image 
A 2D Chem structure

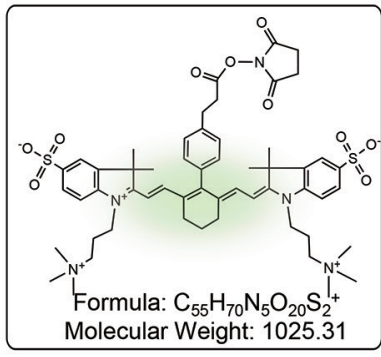

3D Chem structure

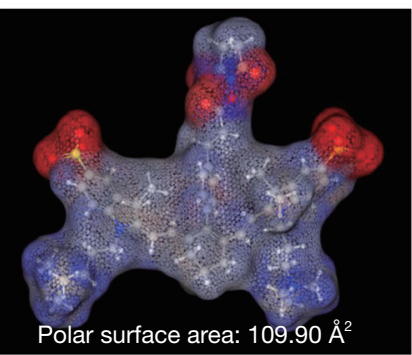

B TLR4-ZW800

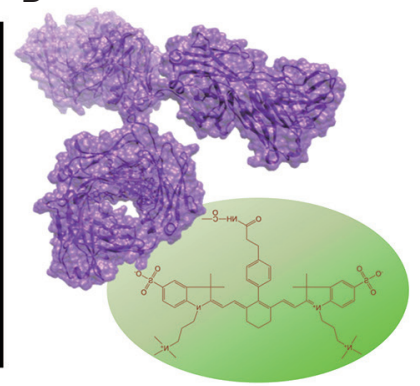

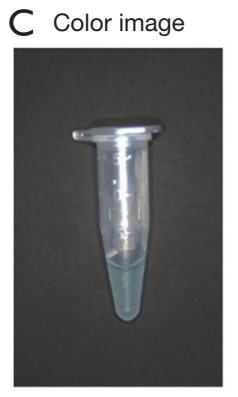

NIR image
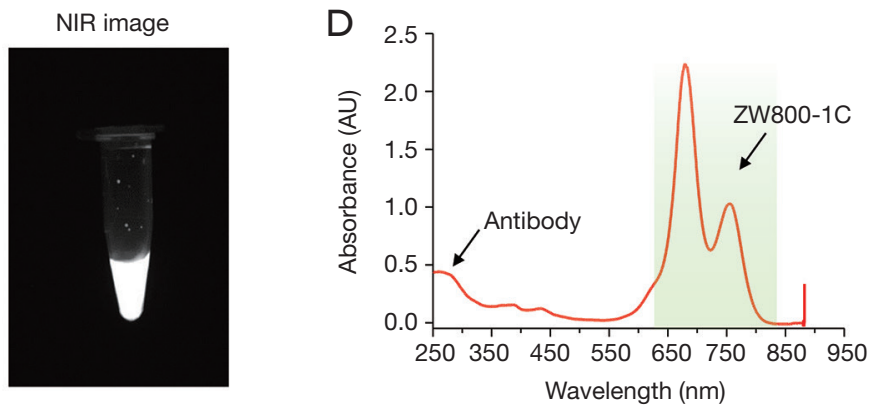

Figure 1 Conjugation of anti-TLR4 antibody with ZW800-1C. ZW800-1 NHS ester (A) was conjugated on anti-TLR4 antibody (B, TLR4-ZW800) in PBS, pH 7.8. The 3D structure of IgG2a antibody is available in the Protein Data Bank (PDB code: 1IGT). (C,D) Optical property of TLR4-ZW800 in 5\% BSA $(10 \mu \mathrm{M})$ under the FLARE imaging system (C) and UV-Vis-NIR spectrophotometer (D). The labeling ratio of ZW800-1 against anti-TLR4 antibody was determined to be 2.47 by using the Beer-Lambert's Law.

analysis using the FLARE imaging system $(12,17,18)$. The blood concentration curve shows that TLR4-ZW800 exhibited a two-compartment profile of in vivo kinetics (Figure 2A). The rapid initial decay of blood concentration was reflected by the efficient initial distribution into capillaries, and the final concentrations at $4 \mathrm{~h}$ postinjection reached close to the baseline representing rapid elimination from the body by the systemic clearance (12). As shown in Figure 2B, the half-life values of TLR4ZW800 are $2.60 \pm 0.77 \mathrm{~min}$ during the distribution phase $\left(t_{1 / 2 \alpha}\right)$, and $72.46 \pm 18.59 \mathrm{~min}$ for the terminal phase $\left(t_{1 / 2 \beta}\right)$. Other pharmacokinetic parameters including plasma clearance $(0.98 \mathrm{~mL} / \mathrm{min})$ and volume of distribution (3.67 mL) of TLR4-ZW800 after a single intravenous injection are summarized in Figure 2C. Urinary excretion of TLR4-ZW800 was also examined under a UV-Vis-NIR spectrophotometer and determined to be $41.62 \% \pm 11.57 \%$ ID (injected dose) at $4 \mathrm{~h}$ post-injection.

We further studied the biodistribution and clearance mechanisms of TLR4-ZW800 in C57BL/6 mice using the FLARE imaging system. The NIR fluorescence signals of TLR4-ZW800 were mainly located in the liver, spleen, and kidneys at 4 h post-injection (Figure $S 2 A$ ). Consistently, signal-to-background ratio (SBR; organs $v s$. muscle) of resected organs was significantly higher in the liver and kidneys (Figure S2B), suggesting that TLR4-ZW800 was removed from blood by myeloid cell compartments in the liver and renal clearance. This is consistent with the known distribution of TLR4 (4), which is expressed by all parenchymal and non-parenchymal cell types in the liver, especially in the Kupffer cells $(19,20)$. Interestingly, the NIR fluorescence signals in the mononuclear phagocyte system (e.g., liver and spleen) were reduced at $24 \mathrm{~h}$ (Figure 3) and $48 \mathrm{~h}$ (Figure S3) post-injection. To examine the distinct contribution of macrophage compartments, we prepared TLR4-ZW800-labeled macrophages and further examined their biodistribution and clearance in C57BL/6 mice (Figure 3). The fluorescence signals in mice injected with the NIR labeled macrophages were mainly trapped in the liver and urinary system at $4 \mathrm{~h}$ (Figure S2C). Furthermore, a higher portion of TLR4ZW800 macrophages were found in the lungs and spleens compared to the biodistribution of TLR4-ZW800 (Figure S2). This is consistent with the known distribution 

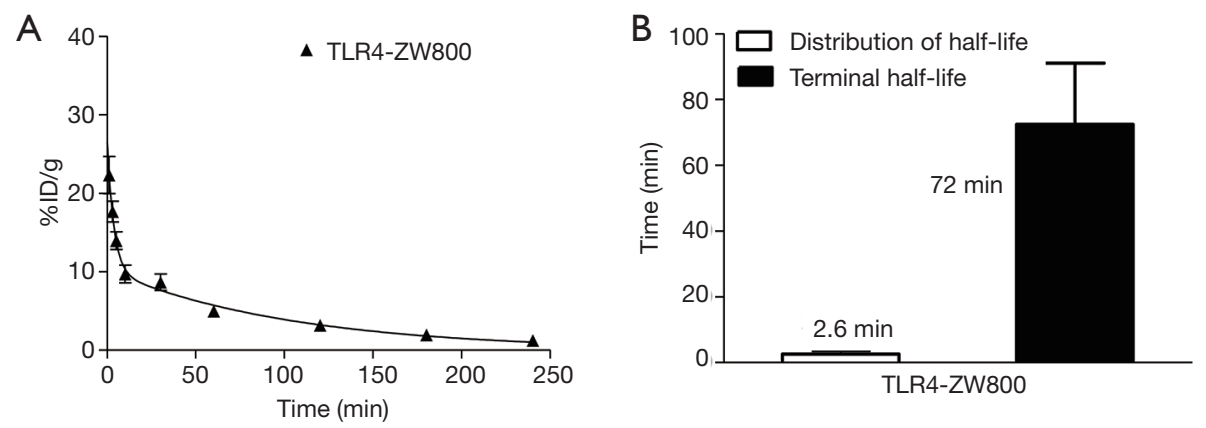

\begin{tabular}{ll}
$\mathrm{C}$ & Pharmacokinetics \\
\cline { 2 - 2 } $\mathrm{T}_{1 / 2} \alpha(\mathrm{min})$ & $2.60 \pm 0.77$ \\
$\mathrm{~T}_{1 / 2} \beta(\mathrm{min})$ & $72.46 \pm 18.59$ \\
$\mathrm{~K}\left(\mathrm{~min}^{-1}\right)$ & 0.0096 \\
$\mathrm{AUC}(\% \mathrm{ID} / \mathrm{g} \cdot \mathrm{min})$ & 1965.00 \\
Plasma clearance $(\mathrm{mL} / \mathrm{min})$ & 0.98 \\
Volume of distribution $(\mathrm{mL})$ & 3.67 \\
\hline Urinary excretion $(\% \mathrm{ID})$ & $4 \mathrm{~h}$ \\
\hline Average & $41.62 \% \mathrm{ID}$ \\
STDEV & $11.57 \% \mathrm{ID}$ \\
\hline
\end{tabular}

Figure 2 Pharmacokinetics of TLR4-ZW800. 10 nmol of TLR4-ZW800 was injected intravenously into C57BL/6 mice 4 h prior to imaging, and their (A) blood concentration (\% ID/g) decay, (B) distribution and elimination half-life values, and (C) pharmacokinetic parameters and urinary excretion were observed $(n=3$, means $\pm S D)$.
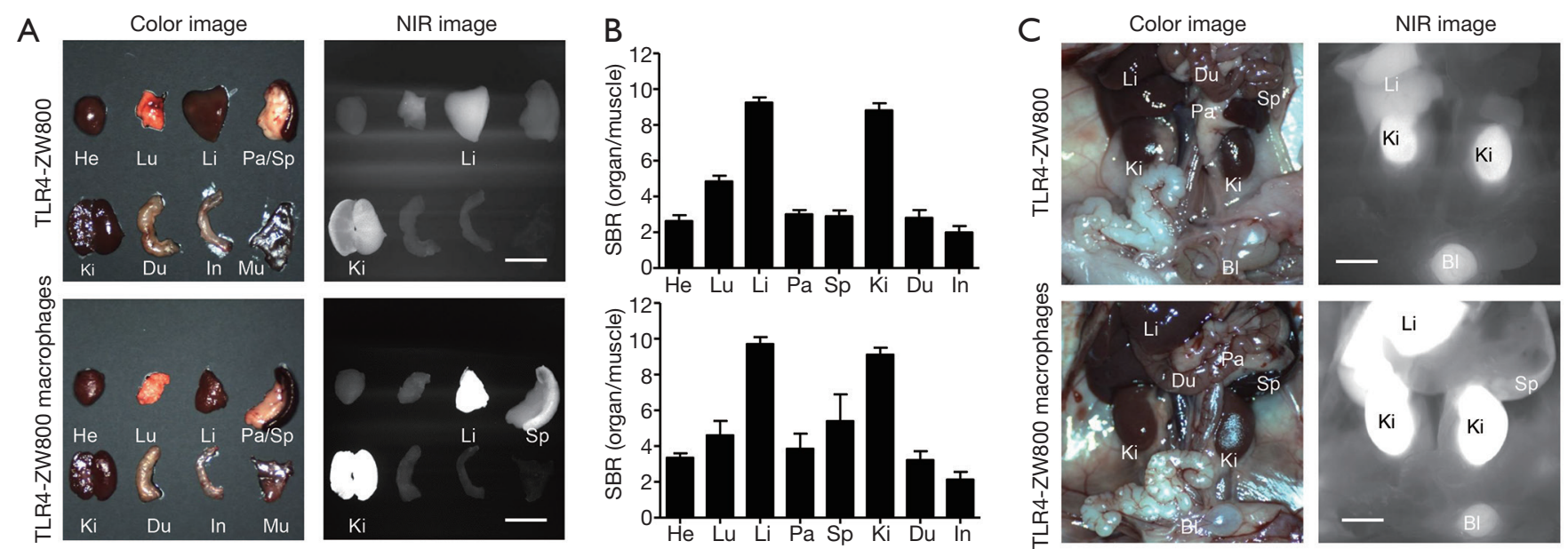

Figure 3 Biodistribution and clearance of TLR4-targeted conjugates at 24 h post-injection. TLR4-ZW800 or TLR4-ZW800-labeled macrophages were injected intravenously into C57BL/6 mice, and their NIR fluorescence signals were observed at $24 \mathrm{~h}$ post-injection under NIR imaging. (A) NIR imaging of resected tissues and organs; (B) signal-to-background ratio (SBR) was calculated by comparing the signals of major organs against surrounding muscle; (C) NIR imaging of abdominal cavity of mice. Scale bars =5 mm. At least 3 mice were analyzed for each sample. Error bars show means \pm SD. Bl, bladder; Du, duodenum; He, Heart; In, intestine; Ki, kidneys; Li, liver; Lu, lungs; Mu, muscle; Pa, pancreas; Sp, spleen.

of macrophages in the mononuclear phagocyte system (21), where liver macrophages and, to a lesser degree, spleen macrophages have been identified as a major factor for the undesirable uptake and clearance of intravenously injected nanomedicines (22). This result indicates that liver is responsible for predominant macrophage uptake and phagocytic activities. Together, TLR4-targeted conjugates are taken up by macrophages in the competing organs (liver) systemically upon intravenous administration, of which biodistribution reflects the localization of TLR4-positive macrophages in vivo.
TAMs are one of the most abundant cell types found in the tumor microenvironment of solid tumors (2). To determine if TLR4-targeting strategy leads to efficient tumor imaging, we injected TLR4-ZW800 intravenously into a syngeneic mouse model of liver cancer and performed real-time intraoperative NIR imaging up to 48 h post-injection (Figures $4, S 3$ ). We observed strong NIR fluorescence signals in the tumor graft after $1 \mathrm{~h}$ post-injection, which increased over the time period of observation. The tumor signal reached a maximum at $48 \mathrm{~h}$ post-injection, suggesting that liver cancer could 

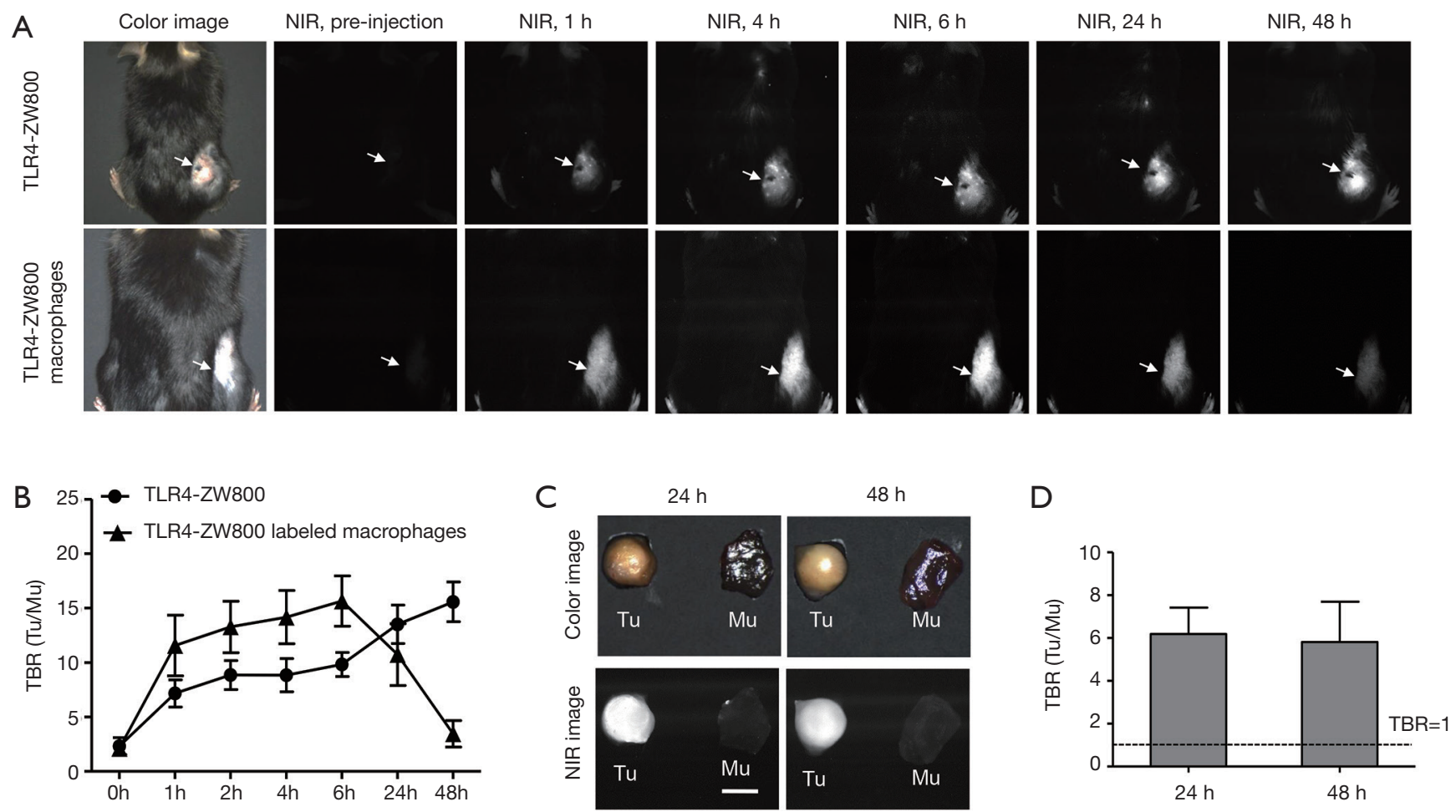

Figure 4 Tumor-targeting with TLR4-ZW800 in a syngeneic mouse model of liver cancer. (A) TLR4-ZW800 (top) or TLR4-ZW800labeled macrophages (bottom) were injected intravenously into tumor-bearing mice, and their NIR fluorescence signals were observed at 1, 2, 4, 6, 24, and 48 h post-injection. Arrows indicate tumor grafts in the flank. (B) Tumor-to-background ratio (TBR) was quantified by comparing the tumor signals against surrounding muscle. (C) NIR imaging and (D) TBR of resected tumors at 24 and $48 \mathrm{~h}$ post-injection of TLR4-ZW800. At least 3 mice were analyzed for each sample. Error bars show means \pm SD. Scale bar =3 mm. Mu, muscle; tu, tumor.

be efficiently targeted using a single bolus injection of TLR4-ZW800. To examine the distinct contribution of macrophage compartments to the signal, we also injected TLR4-ZW800-labeled macrophages into tumor-bearing mice. Interestingly, the fluorescence signal increased up to $6 \mathrm{~h}$ post-injection and gradually decreased over $48 \mathrm{~h}$ of imaging period because of increased uptake in the liver and kidneys (Figure $S 3 C$ ). Then, tumors were resected and imaged under the NIR imaging system (Figure 4C). Consistent with the intraoperative NIR imaging results, fluorescence signals from the resected tumor at 24 and $48 \mathrm{~h}$ post-injection were significantly higher than that of muscle (TBR $\geq 6$ ) (Figure $4 D$ ). We further investigated signal distribution in the resected tumoral tissue under the NIR fluorescence microscope (Figure S4). The signal of resected tumors was predominantly observed in the boundary of tumoral regions at $24 \mathrm{~h}$ post-injection and spread out intratumorally at $48 \mathrm{~h}$ post-injection. This result indicates that the conjugate was taken up by peritumoral region first, which received a relatively ample volume of blood flow than blood flow-poor tumor core (23). Together, these data confirm that molecular targeting TLR4 combined with NIR imaging would be a useful tool for cancer imaging.

\section{Conclusions}

In the study, we demonstrated NIR fluorophore-conjugated anti-TLR4 antibody mediated TAM-specific targeting in a syngeneic mouse model of liver cancer. Since TAMs and TLR4 signaling play a critical role in HCC progression and pathogenesis, our findings suggest that this strategy could be useful for not only cancer diagnosis, but also evaluation of inflammatory milieu of HCC, and, ultimately suppression of tumor progression. Additionally, since macrophage-mediated drug delivery system has been gaining an interest as a novel strategy for cancer treatment $(24,25)$, intraoperative fluorescence imaging can facilitate the diagnosis, prognosis, and treatment of various human 
cancers. This imaging strategy combined with targeted agents might contribute to improve the sensitivity and specificity of the inflammatory status of solid tumors and their prognoses. In conclusion, targeted molecular imaging of TLR4 using a zwitterionic fluorophore ZW800-1C robustly detected TAM-enriched hepatocellular carcinoma after single intravenous administration of the targeted molecular probe under NIR fluorescence imaging. This imaging strategy could be broadly used to detect cancerous tissue with the increased TAM content and evaluate the status of TLR4 signaling in solid tumors.

\section{Acknowledgments}

Funding: Y Ji and Z Wang contributed equally to this work. We thank Ivey Choi for manuscript editing. This study was supported by the US NIH grants NIBIB \#R01-EB022230, NCI \#R21CA223270, and the Creative Materials Discovery Program through the National Research Foundation of Korea (2019M3D1A1078938), Key Research and Development Plan of Shaanxi Province (No. 2017SF-313), and Personnel Training Specialized Research Foundation of the Second Affiliated Hospital of Xi'an Jiaotong University (No. RC(GG)201803).

\section{Footnote}

Conflicts of Interest: The authors have no conflicts of interest to declare.

Ethical Statement: All animal procedures were performed in accordance with the Public Health Service Policy on Humane Care of Laboratory Animals and approved by the MGH IACUC (protocol \#2016N000529).

Disclaimer: The content expressed is solely the responsibility of the authors and do not necessarily represent the official views of the NIH.

\section{References}

1. Ribas A, Wolchok JD. Cancer immunotherapy using checkpoint blockade. Science 2018;359:1350-5.

2. Thorsson V, Gibbs DL, Brown SD, Wolf D, Bortone DS, Ou Yang TH, Porta-Pardo E, Gao GF, Plaisier CL, Eddy JA, Ziv E, Culhane AC, Paull EO, Sivakumar IKA, Gentles AJ, Malhotra R, Farshidfar F, Colaprico A, Parker JS, Mose LE, Vo NS, Liu J, Liu Y, Rader J, Dhankani
V, Reynolds SM, Bowlby R, Califano A, Cherniack AD, Anastassiou D, Bedognetti D, Rao A, Chen K, Krasnitz A, Hu H, Malta TM, Noushmehr H, Pedamallu CS, Bullman S, Ojesina AI, Lamb A, Zhou W, Shen H, Choueiri TK, Weinstein JN, Guinney J, Saltz J, Holt RA, Rabkin CE, Cancer Genome Atlas Research N, Lazar AJ, Serody JS, Demicco EG, Disis ML, Vincent BG, Shmulevich L. The Immune Landscape of Cancer. Immunity 2018;48:812830.e14.

3. Engblom C, Pfirschke C, Pittet MJ. The role of myeloid cells in cancer therapies. Nat Rev Cancer 2016;16:447-62.

4. Vaure C, Liu Y. A comparative review of toll-like receptor 4 expression and functionality in different animal species. Front Immunol 2014;5:316.

5. Li J, Yang F, Wei F, Ren X. The role of toll-like receptor 4 in tumor microenvironment. Oncotarget 2017;8:66656-67.

6. Lopes JA, Borges-Canha M, Pimentel-Nunes P. Innate immunity and hepatocarcinoma: Can toll-like receptors open the door to oncogenesis? World J Hepatol 2016;8:162-82.

7. Yang J, Zhang JX, Wang H, Wang GL, Hu QG, Zheng QC. Hepatocellular carcinoma and macrophage interaction induced tumor immunosuppression via Treg requires TLR4 signaling. World J Gastroenterol 2012;18:2938-47.

8. Yu LX, Yan HX, Liu Q, Yang W, Wu HP, Dong W, Tang L, Lin Y, He YQ, Zou SS, Wang C, Zhang HL, Cao GW, Wu MC, Wang HY. Endotoxin accumulation prevents carcinogen-induced apoptosis and promotes liver tumorigenesis in rodents. Hepatology 2010;52:1322-33.

9. Choi HS, Nasr K, Alyabyev S, Feith D, Lee JH, Kim SH, Ashitate Y, Hyun H, Patonay G, Strekowski L, Henary M, Frangioni JV. Synthesis and in vivo fate of zwitterionic near-infrared fluorophores. Angew Chem Int Ed Engl 2011;50:6258-63.

10. Choi HS, Gibbs SL, Lee JH, Kim SH, Ashitate Y, Liu F, Hyun H, Park G, Xie Y, Bae S, Henary M, Frangioni JV. Targeted zwitterionic near-infrared fluorophores for improved optical imaging. Nat Biotechnol 2013;31:148-53.

11. Hyun H, Owens EA, Narayana L, Wada H, Gravier J, Bao K, Frangioni JV, Choi HS, Henary M. Central C-C Bonding Increases Optical and Chemical Stability of NIR Fluorophores. RSC Adv 2014;4:58762-8.

12. Kang H, Gravier J, Bao K, Wada H, Lee JH, Baek Y, El Fakhri G, Gioux S, Rubin BP, Coll JL, Choi HS. Renal Clearable Organic Nanocarriers for Bioimaging and Drug Delivery. Adv Mater 2016;28:8162-8.

13. Hyun H, Owens EA, Wada H, Levitz A, Park G, Park MH, Frangioni JV, Henary M, Choi HS. Cartilage- 
Specific Near-Infrared Fluorophores for Biomedical Imaging. Angew Chem Int Ed Engl 2015;54:8648-52.

14. Choi HS, Frangioni JV. Nanoparticles for biomedical imaging: fundamentals of clinical translation. Mol Imaging 2010;9:291-310.

15. Hu S, Kang H, Baek Y, El Fakhri G, Kuang A, Choi HS. Real-Time Imaging of Brain Tumor for Image-Guided Surgery. Adv Healthc Mater 2018;7:e1800066.

16. Matsuguchi T, Musikacharoen T, Ogawa T, Yoshikai Y. Gene expressions of Toll-like receptor 2, but not Toll-like receptor 4 , is induced by LPS and inflammatory cytokines in mouse macrophages. J Immunol 2000;165:5767-72.

17. Choi HS, Liu W, Liu F, Nasr K, Misra P, Bawendi MG, Frangioni JV. Design considerations for tumour-targeted nanoparticles. Nat Nanotechnol 2010;5:42-7.

18. Chung JE, Tan S, Gao SJ, Yongvongsoontorn N, Kim SH, Lee JH, Choi HS, Yano H, Zhuo L, Kurisawa M, Ying JY. Self-assembled micellar nanocomplexes comprising green tea catechin derivatives and protein drugs for cancer therapy. Nat Nanotechnol 2014;9:907-12.

19. Rivera CA, Adegboyega P, van Rooijen N, Tagalicud A, Allman M, Wallace M. Toll-like receptor-4 signaling and Kupffer cells play pivotal roles in the pathogenesis of nonalcoholic steatohepatitis. J Hepatol 2007;47:571-9.

20. Tsung A, Hoffman RA, Izuishi K, Critchlow ND, Nakao A, Chan MH, Lotze MT, Geller DA, Billiar TR.

Cite this article as: Ji Y, Wang Z, Bao K, Park GK, Kang H, Hu S, McDonald E, Kim MS, Kashiwagi S, Choi HS. Targeted molecular imaging of TLR4 in hepatocellular carcinoma using Zwitterionic near-infrared fluorophores. Quant Imaging Med Surg 2019;9(9):1548-1555. doi: 10.21037/qims.2019.09.04
Hepatic ischemia/reperfusion injury involves functional TLR4 signaling in nonparenchymal cells. J Immunol 2005;175:7661-8.

21. Gordon S, Pluddemann A, Martinez Estrada F. Macrophage heterogeneity in tissues: phenotypic diversity and functions. Immunol Rev 2014;262:36-55.

22. Andón FT Digifico E, Maeda A, Erreni M, Mantovani A, Alonso MJ, Allavena P. Targeting tumor associated macrophages: The new challenge for nanomedicine. Semin Immunol 2017;34:103-13.

23. Martin JD, Fukumura D, Duda DG, Boucher Y, Jain RK. Reengineering the Tumor Microenvironment to Alleviate Hypoxia and Overcome Cancer Heterogeneity. Cold Spring Harb Perspect Med 2016. doi: 10.1101/ cshperspect.a027094.

24. Pang L, Qin J, Han L, Zhao W, Liang J, Xie Z, Yang P, Wang J. Exploiting macrophages as targeted carrier to guide nanoparticles into glioma. Oncotarget 2016;7:37081-91.

25. Huang WC, Chiang WH, Cheng YH, Lin WC, Yu CF, Yen CY, Yeh CK, Chern CS, Chiang CS, Chiu HC. Tumortropic monocyte-mediated delivery of echogenic polymer bubbles and therapeutic vesicles for chemotherapy of tumor hypoxia. Biomaterials 2015;71:71-83. 


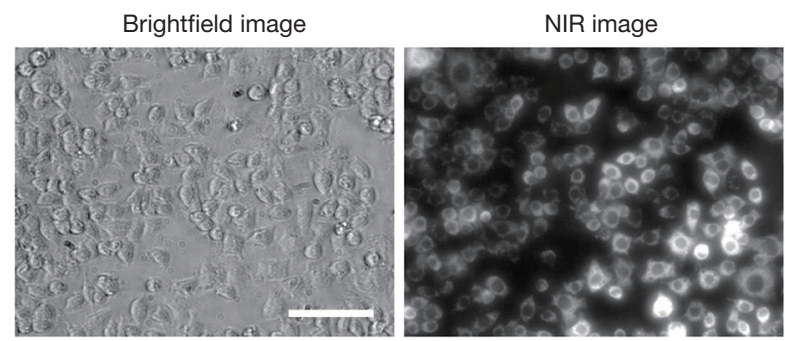

Figure S1 In vivo evaluation of the targetability of the TLR4-ZW800 conjugate. The binding of TLR4-ZW800 to TLR4 was evaluated using mouse RAW264.7 cells in culture. The NIR fluorescent signals were found in the cytosol of RAW264.7 cells, indicating the involvement of receptor-mediated endocytosis. Scale bar $=100 \mu \mathrm{m}$.
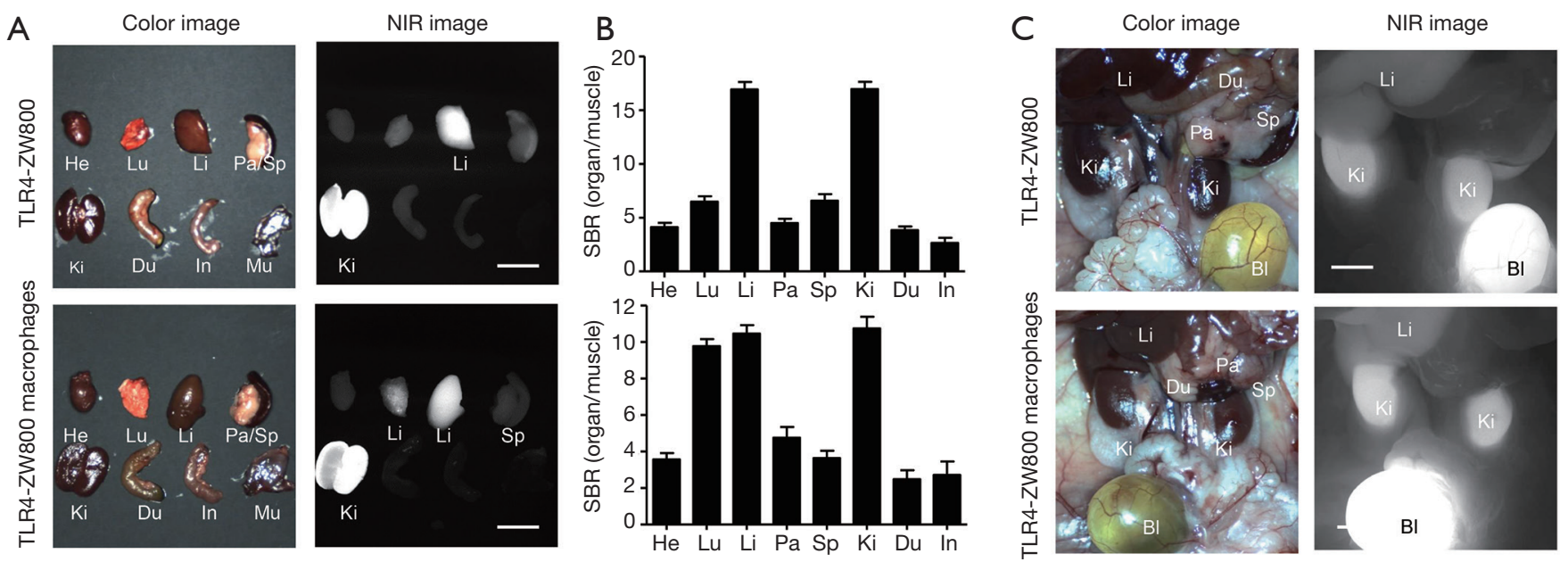

Figure S2 In vivo biodistribution and clearance of the TLR4-targeted conjugates at 4 h post-injection. TLR4-ZW800 or TLR4-ZW800labeled macrophages were injected intravenously into C57BL/6 mice, and their NIR fluorescence signals were observed at 4 h postinjection. (A) NIR imaging of resected major tissues and organs; (B) signal-to-background ratio (SBR) was calculated by comparing the signals of resected tissue against muscle; (C) intraoperative NIR imaging of abdominal cavity of mice. At least 3 mice were analyzed for each sample. Error bars show means \pm SD. Bl, bladder; Du, duodenum; He, Heart; In, intestine; Ki, kidneys; Li, liver; Lu, lungs; Mu, muscle; Pa, pancreas; Sp, spleen. 

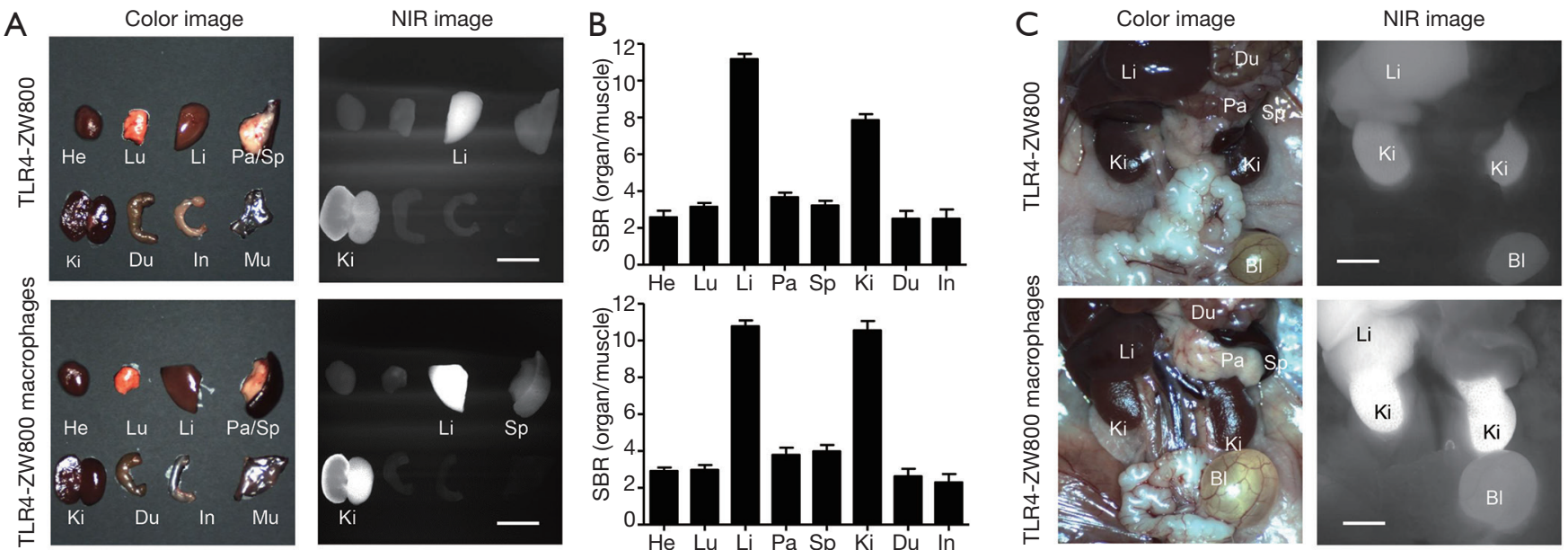

Figure S3 In vivo biodistribution and clearance of the TLR4-targeted conjugates at 48 h post-injection. TLR4-ZW800 or TLR4-ZW800labeled macrophages were injected intravenously into C57BL/6 mice, and their NIR fluorescence signals were observed at 48 h postinjection. (A) NIR imaging of resected major tissues and organs; (B) SBR was calculated by comparing the signals of the resected major tissue against muscle; (C) intraoperative NIR imaging of abdominal cavity of mice. At least 3 mice were analyzed for each sample. Error bars show means \pm SD. Abbreviations used are: Bl, bladder; Du, duodenum; He, Heart; In, intestine; Ki, kidneys; Li, liver; Lu, lungs; Mu, muscle; Pa, pancreas; Sp, spleen.
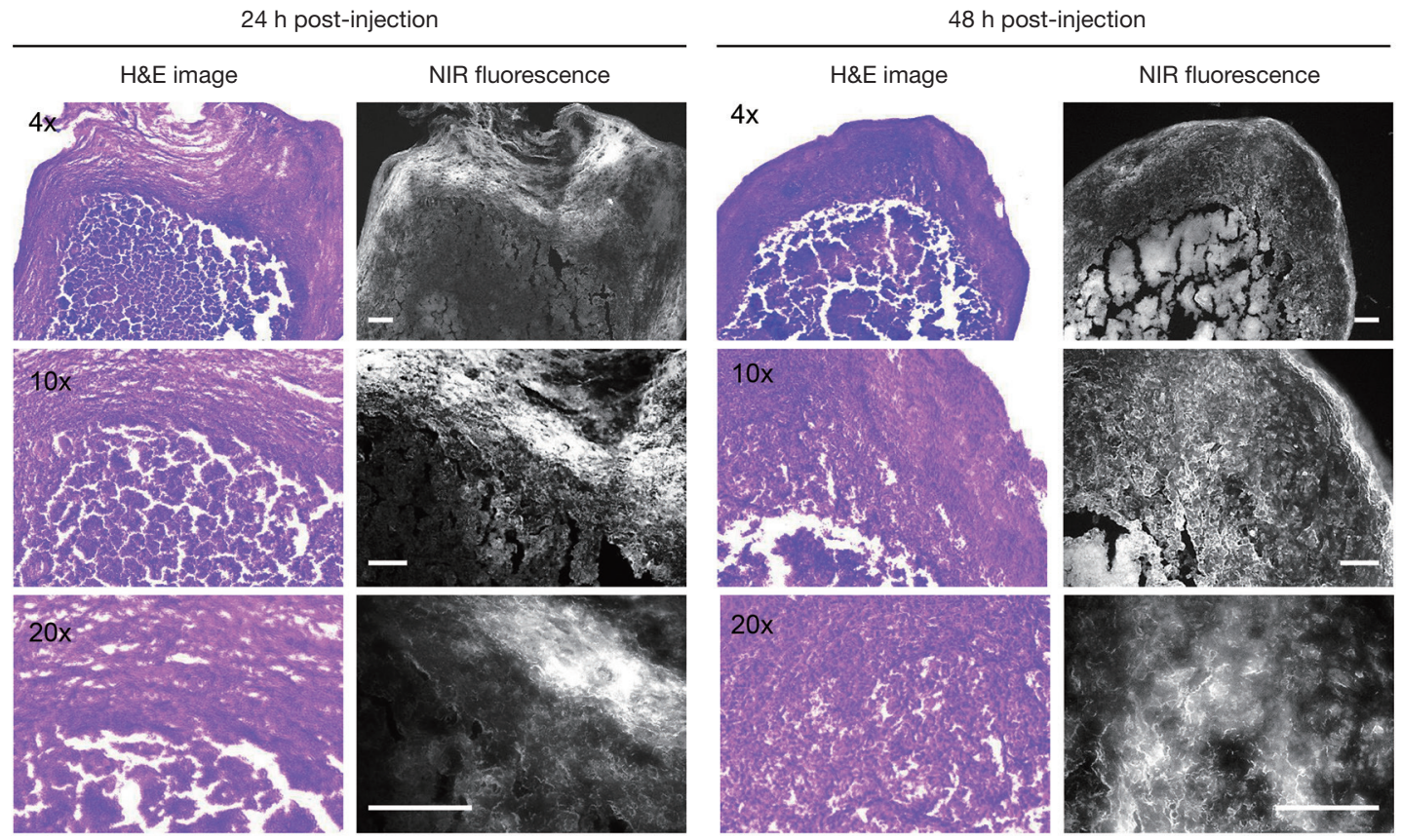

Figure S4 Histological analysis of uptake of the TLR4-targeted conjugates in the tumor. TLR4-ZW800 was injected intravenously into tumor-bearing mice 24 and $48 \mathrm{~h}$ prior to resection. H\&E staining and NIR fluorescence of resected tumors were imaged under the multichannel NIR fluorescence microscope. Scale bar $=200 \mu \mathrm{m}$. 